\title{
Germanica
}

\section{Le livret de Lulu d'Alban Berg: crise du sens et interrogation sur l'art}

Das Libretto von Alban Bergs Lulu : Bedeutungskrise und Frage nach der Kunst

\section{Fabrice Malkani}

\section{OpenEdition}

\section{Journals}

Édition électronique

URL : http://journals.openedition.org/germanica/490

DOI : 10.4000/germanica.490

ISSN : $2107-0784$

Éditeur

Université de Lille

Édition imprimée

Date de publication : 1 décembre 2007

Pagination : 73-90

ISBN : 978-2-913857-20-9

ISSN : 0984-2632

Référence électronique

Fabrice Malkani, « Le livret de Lulu d'Alban Berg: crise du sens et interrogation sur l'art », Germanica [En ligne], 41 | 2007, mis en ligne le 01 décembre 2009, consulté le 10 décembre 2020. URL : http:// journals.openedition.org/germanica/490 ; DOI : https://doi.org/10.4000/germanica.490

Ce document a été généré automatiquement le 10 décembre 2020.

(c) Tous droits réservés 


\title{
Le livret de Lulu d'Alban Berg: crise $\mathrm{du}$ sens et interrogation sur l'art
}

Das Libretto von Alban Bergs Lulu : Bedeutungskrise und Frage nach der Kunst

\author{
Fabrice Malkani
}

1 Portant le seul titre de Lulu, l'opéra d'Alban Berg (1885-1935) reprend en un texte unique et cohérent les arguments de deux pièces de Frank Wedekind (1864-1918), Erdgeist (L'Esprit de la terre, 1895) et Die Büchse der Pandora (La Boîte de Pandore, 1902) ${ }^{1}$. Si Alban Berg compose sa musique sur ce canevas emprunté à Wedekind, il est également l'auteur du livret complexe qui réorganise complètement la matière des deux pièces dont il conserve des passages entiers tout en modifiant considérablement l'agencement de certaines scènes ${ }^{2}$. Comme l'auteur dramatique allemand, le compositeur jette un regard critique sur la société et sur ses contemporains et les invite à prendre conscience de leurs travers et de leurs contradictions. En relativisant la portée des bienfaits de la "modernité ", dont il dénonce à l'inverse les conséquences néfastes et mortifères, il utilise les procédés de la caricature et de la satire, dans un propos où le grotesque alterne avec la provocation, tout en maintenant chez les personnages principaux une dimension humaine qui rend leurs sentiments crédibles. C'est le conflit profond entre l'image de soi et la nature indomptée qui nous est présenté, comme si la modernité conduisait à une forme aggravée de l'opposition classique entre la loi et la liberté que l'intériorisation de la loi permettait naguère encore de surmonter. Il appartiendra à une femme, Lulu, d'être le révélateur de ce que l'on peut qualifier de crise de la modernité. C'est par elle que la vérité adviendra, autant que le scandale, la destruction et la mort. L'opéra Lulu apparaît comme la métaphore d'un monde autodestructeur, fondé sur des rapports de force, de possession, de rivalité et de jalousie. En réduisant les sept actes et deux prologues des deux pièces de Wedekind en trois actes et un prologue, Berg concentre l'action et accentue les contrastes sans jamais réduire le contenu idéel ni effacer les références implicites aux analyses contemporaines de la société. 


\section{Le tableau d'une époque ou la « tragédie de la culture »}

2 À la manière de Rosa Fröhlich, l'héroïne du roman de Heinrich Mann (1871-1950), Professor Unrat oder das Ende eines Tyrannen (Professeur Ordure ou la fin d'un tyran, 1905) rendu célèbre par le film de Sternberg, L’Ange bleu (1930), Lulu séduit les hommes dont elle démasque les pulsions violentes, possessives et dominatrices, les conduisant à la déchéance sociale et au suicide ou servant d'instrument à leur suicide, comme dans le cas du Docteur Schön ${ }^{3}$. Objet de toutes les convoitises, projection de tous les désirs masculins, Lulu se voit reprocher d'être volage. Mais en réponse à ces reproches, elle déclare, de manière paradoxale et profonde à la fois: "Je n'ai jamais voulu paraître autre chose en ce monde que ce pour quoi l'on m'a prise. Et l'on ne m'a jamais prise en ce monde pour autre chose que ce que je suis» (fin de la Chanson de Lulu, II, 1) . $^{4}$ L'innocence de Lulu s'exprime par l'ignorance qu'elle oppose aux questions du peintre (I, 1: «Es-tu capable de dire la vérité ?», etc.), répétant jusqu'à sept fois les mots " Je ne sais pas $»^{5}$. Simultanément, cette septuple affirmation révèle une crise du sens liée au contexte d'une modernité ressentie comme une perte des repères et une fuite en avant.

3 La problématique du rapport entre les sexes, liée à la représentation d'une société fondée sur le profit et obsédée par la soif de posséder, où tout se dévalue rapidement, à l'image des actions de la Jungfrau (dont le nom parlant signifie « vierge ») dans l'acte III (scène 1), montre l'actualité des thèmes traités par Wedekind et par Berg dans la mouvance expressionniste et contestataire qui rejoint, par son analyse sociale, certains aspects des essais du philosophe et sociologue allemand Georg Simmel (1858-1918) ${ }^{6}$, rédigés entre 1914 et 1992 et réunis dans leur traduction française sous le titre de Philosophie de la modernité ${ }^{7}$. Ce philosophe est également l'auteur, entre autres, d'une Philosophie de l'argent (1900) ainsi que d'ouvrages consacrés à la problématique de l'individu et de la société et à la philosophie de l'art ${ }^{8}$. Il ne s'agit pas ici de chercher à savoir ce que Wedekind ou Berg pourraient devoir à Simmel, mais de constater une convergence d'analyses, une similitude de vues à travers des discours différents (philosophique, théâtral, lyrique et musical). La scène lyrique est aussi un lieu d'expression privilégié de l'histoire des idées.

4 Simmel dénonce la subordination de la qualité à la quantité, la transformation des valeurs en marchandises, dans ce qu'il appelle la «tragédie de la culture "9. À partir de la distinction classique entre la "femme-nature et "l'homme-culture», il attribue l'opposition et l'incompréhension entre hommes et femmes principalement à la dépersonnalisation de l'homme liée à l'avènement de la grande ville et paradoxalement inséparable du besoin de se distinguer. La superficialité des rapports humains, la légèreté tant prisée des années vingt du vingtième siècle - les années dorées, "die goldenen Zwanziger» - sont pour lui la cause de l'émergence des forces destructrices issues des strates les plus profondes de l'être. Parallèlement, Simmel analyse le lien entre l'argent et la féminité: l'attrait (der Reiz) de l'argent et celui de la femme se ressemblent (reizend signifie "charmante »), ils évoquent tous deux la possession d'un bien, «la promesse d'une jouissance ». Dans un essai préliminaire intitulé Le rôle de l'argent dans les rapports entre les sexes, le philosophe étudie l'histoire de l'achat des femmes en vue du mariage et le lien qui s'établit avec la prostitution ${ }^{10}$.

Lulu délivre un message semblable. Le thème de la prostitution parcourt toute l'œuvre et s'exprime sans détour dans la dernière scène, située symboliquement à Londres, modèle de la grande ville moderne où la prostitution est liée au développement de 
l'urbanisation et de l'industrialisation. Comme Lulu dès le Prologue, la figure de la prostituée suscite fascination et répulsion: séductrice et destructrice, elle est finalement détruite - ou plus exactement, elle se détruit elle-même.

\section{Représentations de la femme et rôle de l'argent}

6 Les autres représentations de femmes dans l'opéra s'inscrivent dans le droit fil de ces considérations: la décoratrice de l'acte III (scène 1) porte "une robe à la mode ${ }^{11}$ et déclare à l'Athlète qu'elle " préfère les acrobates ${ }^{12}$; la jeune fille de quinze ans, objet de la concupiscence des hommes présents, est appelée "petite princesse $»^{13}$; sa mère songe surtout aux actions de la Jungfrau. Les seules femmes qui s'opposent au rôle dans lequel la société veut les confiner et à l'aliénation qui en résulte sont Lulu, refusant avec véhémence la condition de prostituée à laquelle veut la réduire le Marquis (III, 1): "Je ne suis pas faite pour ce métier $»^{14}$, et la comtesse Geschwitz qui, juste avant d'être assassinée par Jack l'Éventreur, déclare: «Je m’inscrirai à l'université. Il faut que je lutte pour les droits des femmes, que j'étudie le droit» (III, 2) ${ }^{15}$. Plus qu'un féminisme (que Wedekind rejetait, y voyant la perspective d'une simple imitation des hommes par les femmes), ces mots, de même que le rapport qui s'établit entre Lulu et la comtesse, traduisent la volonté de faire valoir l'identité féminine dans sa dimension propre: c'est ce que l'on appelle à l'époque la philogynie, un amour de la femme qui serait aussi désintéressé que l'est l'amour de la sagesse ou philosophie.

Or l'intérêt matériel domine dans le monde ici dépeint, qui est celui des hommes. L'argent joue un rôle essentiel dans tout l'opéra, depuis le prix du tableau - le profit que le peintre tire du modèle qu'il épousera ensuite (ce qui fait dire à Schön, à trois reprises: « Tu as épousé un demi-million » I, ${ }^{16}$ ) jusqu'au coût du spectacle, en passant par la mendicité de Schigolch, l'intérêt de Schön pour la Bourse, le rôle du Banquier dans l'acte III, le cours des actions qui monte et puis s'effondre, le dénuement matériel de Lulu et les exigences de l'Athlète, puis du Marquis prêt à prostituer Lulu pour mille deux cents marks, la mise à prix de la tête de Lulu (la prime de la police pour l'arrestation du meurtrier de Schön), le dénuement de la comtesse Geschwitz conservant malgré tout la toile du portrait, l'argent que Lulu (ou Alwa) attend de la prostitution. C'est aussi le soupçon de se voir voler son argent qui conduit le Nègre qui s'était refusé à montrer sa pièce d'or avant d'entrer avec Lulu - à tuer Alwa, tandis que Jack récupère la pièce qu'il avait tout d'abord donnée à Lulu. Toute l'action est ainsi rythmée, structurée par la présence de l'argent, monnaie d'échange et échelle de valeurs matérielles.

8 Par deux fois cependant, la préoccupation financière entre en contradiction flagrante avec la logique du désir amoureux: lorsque le peintre s'arrache à l'étreinte qu'il avait obtenue de Lulu pour répondre à un coup de sonnette, en arguant qu'il s'agit « peutêtre du marchand d'art ", alors que celle-ci tente de l'en dissuader (I, 2) ${ }^{17}$; lorsque Schön, à Lulu qui lui demande s'il ne pourrait pas se libérer l'après-midi pour sortir en voiture avec elle, répond qu'il doit aller à la Bourse (II, 1$)^{18}$. Dans les deux cas, Lulu voit son époux placer les affaires financières avant les sentiments amoureux ou conjugaux, selon une hiérarchisation propre à la société à laquelle ils appartiennent. La répétition de cette situation illustre la dépersonnalisation des hommes et le modèle engendré par une structure sociale aliénante. 


\section{Le cirque et la ménagerie}

9 D'emblée, l'opéra nous présente le monde comme une collection d'espèces animales enfermées dans un cirque. La ménagerie du prologue est le lieu de captivité et d'exposition des instincts vitaux qui se manifesteront ensuite tout au long de l'action ; le dompteur apparaît comme une allégorie de la civilisation, avec ses vêtements impeccables, son fouet et son revolver. La société elle-même est un cirque, dont le premier représentant sur scène est un clown. Or la figure du saltimbanque est en soi une mise en cause de l'honorabilité bourgeoise. On ne saurait oublier que Wedekind s'est inspiré de la reprise, en 1892, d'une pantomime de Félicien Champsaur, Lulu, créée au Nouveau Cirque en 1888 et constituant le canevas du texte de Champsaur de 1901, Lulu. Roman clownesque ${ }^{19}$. La musique de cirque du Prologue reviendra d'ailleurs au début de l'acte III.

10 Toutes les dimensions essentielles de cet opéra sont ainsi présentes dès le Prologue: le règne de la mascarade, l'emprisonnement de la spontanéité, l'asservissement des pulsions élémentaires, le primat de l'apparence et du faux-semblant, l'étalage du pouvoir et de la violence. Les paroles du dompteur expriment la dichotomie entre l'instinct et l'esprit qui, dans le discours théorique de l'époque, correspond étroitement à la distinction homme-femme: à l'opposition entre la « fierté » des hommes et la « joie de vivre » des femmes répond la subordination de la « créature » au " génie » ${ }^{20}$.

11 Mais par le truchement du dompteur, l'auteur nous promet autre chose que le spectacle convenu de la nature civilisée tel que le théâtre jusqu'ici nous l'a montré. En annonçant l'entrée en scène de la Bête, il se propose de montrer le Vrai, quelle que soit sa sauvagerie: derrière chacun des animaux énumérés (le tigre, l'ours, le singe, le chameau, etc.), l'on reconnaîtra les personnages de l'opéra comme autant de variations sur un thème unique. Dans son propos comme dans son écriture, Lulu est bien une œuvre « kaléidoscopique $»^{21}$, nous invitant à la fois à contempler la diversité des formes et des apparences et à nous interroger sur la manière dont la société, ici mise au banc des accusés, façonne une attitude identique pour chacun de ces êtres, tous des hommes, à l'exception du rôle-titre, Lulu, et de la singulière comtesse Geschwitz (est-elle le diable, comme le prétendent Alwa et le lycéen, cette femme qui, avant de mourir, appelle Lulu «mon ange ${ }^{22}$, alors que Schön ne voyait en elle qu'un ange exterminateur $^{23}$ ?). Le dompteur s'interrompt d'ailleurs dans son énumération pour interpeller un machiniste en lui réclamant le serpent, associé dans l'imaginaire judéochrétien à l'image de la femme séductrice et cause du péché originel. Le propos est outré jusqu'à la caricature (et ce n'est pas un hasard si le machiniste "ventripotent " répond à un prénom de clown, Auguste, dont la particularité est de porter un maquillage violent et caricatural).

\section{La confusion des valeurs: de la femme montrée à la femme monstre}

La dimension démonstrative ressortit à la fois à la provocation et, paradoxalement, à une forme de captatio benevolentiae puisqu'il s'agit de relativiser d'avance la crudité du propos et les excès de débauche, de libertinage et de représentation de la sexualité à venir. C'est dans le sens d'une sorte de confusion des valeurs et d'exposé des 
contradictions d'une société ici tournée en ridicule qu'il faut comprendre la juxtaposition d'un amour éthéré («Douce innocence, mon plus grand trésor ! ${ }^{24}$ ) et d'une frayeur séculaire («Elle fut créée pour causer le malheur, / Pour attirer, pour séduire et pour empoisonner, / - Et pour assassiner, sans que quiconque ne s'en aperçoive $»^{25}$ ). Cette femme, ainsi porteuse de tous les péchés, est aussi qualifiée de « douce bête » à qui le Dompteur demande de ne pas déformer « la figure originale » ou « originelle» (Urgestalt) - de la Femme. C'est ainsi qu'il commande à l'Auguste de la remettre à sa place ${ }^{26}$. Les deux hommes font de Lulu un objet, apporté, exposé, caressé, montré du doigt, remporté.

13 Sous le travestissement masculin, c'est Ève qui nous est présentée, ou Pandore, ou encore Lilith, dont le nom est phonétiquement le plus proche de Lulu: créée selon la tradition kabbalistique directement de la terre (et non d'une côte d'Adam), Lilith passe pour une première Ève, qui fut cependant l'égale d'Adam. À la suite d'une dispute avec ce dernier, elle s'enfuit pour mener une carrière démoniaque. Elle dut ensuite être maintenue dans les profondeurs de la mer afin de ne pas nuire aux humains, car elle était devenue l'ennemie des amours conjugales et l'instigatrice d'amours interdites. Devenue ainsi la rivale d'Ève, elle tenta de séduire Adam et fut pour cette raison rejetée dans l'abîme.

14 Le décalage ici s'opère à plusieurs niveaux: Lulu, travestie en homme, est déguisée en clown ; assassin et animal à la fois, elle doit incarner simultanément un éternel féminin confiné dans la représentation d'une virginité dont l'homme (le dompteur) se rend propriétaire et qu'il détient comme une richesse monnayable.

15 La femme montrée sera la femme monstre: il est permis de répondre ainsi à la question du dompteur au public lorsqu'il s'apprête, dit-il, à mettre sa tête entre les dents d'un fauve, en ouvrant alors le rideau sur le premier $\operatorname{acte}^{27}$. On peut rappeler ici que la pièce de Wedekind, La Boite de Pandore, portait dans la première version (1895) le sous-titre de Eine Monstretragödie - tragédie d'un monstre ou tragédie monstrueuse -, rappelant la double fonction du monstre qui est de montrer - ou d'être montré - et d'effrayer. C'est également la fonction de cet opéra, portant sur la scène lyrique la volonté de représentation du laid, voire de l'obscène, dans le but de guérir par un choc salutaire une société corrompue et décadente, et d'exprimer le vrai sans détour ni voile pudique tendu au nom d'une esthétique dissimulatrice. On peut aussi - c'est l'interprétation commune - comprendre que le fauve en question n'est autre que le public lui-même auquel l'artiste livre son œuvre, le dompteur étant dès lors identifié à l'auteurcompositeur.

\section{Réflexion sur l'art}

Cet opéra qui met en scène, entre autres, un peintre, une actrice et un compositeur, comporte en son centre un film ${ }^{28}$, insérant dans le triple discours de l'opéra (littéraire, musical et scénique) la technique moderne de l'image animée, comme une réflexion sur la problématique de l'art et sur la modification de ses supports et de sa perception par le biais de procédés nouveaux, alors en plein développement ${ }^{29}$.

17 Cette dimension réflexive - la mise en scène de l'artiste -, est évidente dès la première scène du premier acte, où une double présentation de l'art crée une sorte de mise en abyme de la réflexion esthétique. Les premiers mots sont prononcés par un 
compositeur (Alwa ${ }^{30}$ ) qui entre dans l'atelier d'un peintre: «Puis-je entrer ? $»^{31}$. On a fait observer que cette réplique, qui se trouve déjà chez Wedekind, permet d'identifier Alwa à l'auteur ou au compositeur ${ }^{32}$, demandant ainsi la permission d'entrer dans son œuvre même. Redoublement du seuil entre la réalité et le spectacle peut-être, effet de distanciation sans doute, manière en tout cas d'inviter le public à entrer dans cet opéra à sa suite (comme nous y incitait le dompteur du Prologue) afin de jeter sur le monde un regard neuf. Manière aussi de distinguer entre l'art et la réalité puisque le peintre, dont les indications scéniques redoublent la fonction («Le peintre devant le chevalet, peignant $»^{33}$ ), fait de Lulu un portrait en Pierrot qui deviendra en quelque sorte "l'image originale" (Urgestalt) (celle que le dompteur conjurait Lulu de ne pas déformer ${ }^{34}$ ) et qui seule survivra jusqu'à la fin de l'opéra (et au-delà), après les métamorphoses et avanies successives de Lulu et des autres personnages.

C'est toujours en costume de Pierrot que le spectateur découvre Lulu au début du premier acte. D'emblée, Lulu est donc la représentation de ce que les hommes font d'elle, puisque c'est son portrait en Pierrot - en clown? - que réalise le peintre sous l'œil du Docteur Schön, puis de son fils Alwa. Or Pierrot, dans la commedia dell'arte, est un personnage naïf et gauche, en tout cas inoffensif - ce serait donc ici l'image que les hommes se font de la femme -, à l'origine de nombreux quiproquos, et qui souvent se déguise en femme. D'une certaine manière, nous avons affaire ici à un retournement: car Lulu déguisée en Pierrot est une femme déguisée en homme..$^{35}$

Toutefois, ce n'est que dans l'imaginaire du peintre - et des hommes de la pièce - que Lulu joue la comédie. Ne s'exclame-t-elle pas, lorsque le peintre, confondant la séduction de l'art avec celle de la vraie femme qu'est Lulu, lui déclare son amour et exige le tutoiement: «Moi, jouer la comédie? Je n'en ai jamais eu besoin $»^{36}$. Au reste, le peintre, censé voir le monde et les êtres pour les représenter, est finalement aveugle: «Il ne voit rien. Il ne me voit pas et il ne se voit pas lui-même. Il est aveugle, aveugle, aveugle... », déclare Lulu à deux reprises $(\mathrm{I}, 2)^{37}$.

Si le peintre ensuite l'appelle Nelly, puis décide de la nommer Eva, c'est bien qu'il projette en Lulu sa propre image d'une femme qu'elle n'est pas, comme elle le lui fait remarquer: «Je ne m'appelle pas Nelly, je m'appelle Lulu »38. Quant à Schön, et Alwa à sa suite, ils l'appellent Mignon. "J'ignore comment elle s'appelait en réalité » (I, 2) ${ }^{39}$. Lulu l'innommable ou la femme sans nom? Lorsque Schigolch l'appelle Lulu, elle s'exclame: «Dire que tu m'appelles Lulu [...] Il y a une éternité que je ne m'appelle plus Lulu» $(\mathrm{I}, 2)^{40}$. Et elle ajoute: «Je ne suis plus... qu'une bête» (ein Tier). On apprend d'ailleurs au cours de l'opéra que Lulu n'a ni mère ni père officiellement identifiés (un doute plane sur l'identité réelle de Schigolch qui pourrait être son père, cf. II, 1).

\section{Fonction symbolique du portrait de Lulu: de l'atelier au cadre doré}

21 Le destin du tableau de Lulu en Pierrot retrace la place de l'art dans l'évolution de la société qui nous est montrée sur scène: tout d'abord il passe très logiquement de l'atelier du peintre - où il conquiert, par sa juxtaposition avec son modèle vivant, à la fois le Dr Schön et son fils Alwa - au «salon très élégant » de la deuxième scène où il trône au-dessus de la cheminée dans un "somptueux cadre de brocart $»^{41}$ (par contraste, Lulu est en peignoir et se regarde dans un miroir - le cadre du tableau signifie déjà sa coupure par rapport au modèle réel et son insertion dans un contexte 
où il prend une autre signification). Mais dans la troisième scène, ce n'est plus qu'une copie du tableau que l'on voit sur une grande affiche, recouverte d'inscriptions qui la masquent en partie (tandis que Lulu est «invisible, derrière un paravent $»^{42}$ ). Cette dégradation - copie de la copie -, recouverte de graffitis, évoque précisément le destin de l'art et son statut dans la société. Alwa, le compositeur, contemplant l'affiche, exprime le point de vue de l'artiste dans le processus de création de l'opéra auquel nous sommes en train d'assister:

Ma foi, elle ferait un sujet d'opéra intéressant. (devant l'affiche) Scène un: le professeur de médecine... Ça commence mal! (on entend, venant de l'extérieur, très atténués, des applaudissements et des bravos soutenus) Quel tapage! Comme dans la ménagerie au moment où les fauves voient arriver la nourriture devant la cage. (⿳亠㐅子 nouveau devant le tableau) Scène deux: le peintre... Encore pire ! - Scène trois: faut-il vraiment que ça continue ? ${ }^{43}$

Quant au prince, il ne parle de Lulu que par l'intermédiaire du tableau auquel il se réfère en désignant l'affiche ${ }^{44}$.

Dans la première scène de l'acte II, le portrait, « dans un cadre à l'ancienne doré »" posé sur un chevalet, dans une sorte de mise en scène de l'acte artistique (Lulu, en peignoir, se trouvant dans un fauteuil) et fait l'admiration de la comtesse Geschwitz ( " c'est comme dans un conte $»^{46}$ ) tandis que Lulu évoque l'aversion de son mari pour ce portrait. Les mots de la comtesse Geschwitz retiennent l'attention, car la phrase allemande dit textuellement: «Là, vous êtes comme un conte.» Cette représentation de la femme n'est-elle qu'illusion, fable, légende ? C'est que la comtesse évoque le bal des femmes artistes et demande à Lulu d'y paraître en costume masculin, associant ainsi le déguisement de Pierrot à un travestissement qu'elle a elle-même adopté dans sa vie quotidienne, comme nous l'indiquent les didascalies. On notera d'ailleurs que Lulu est amenée par deux fois à travestir son identité: elle prendra les vêtements de la comtesse pour sortir de prison (II, 2), puis ceux du groom pour échapper au commissaire (III, 2).

Dans la deuxième scène de l'acte II, le chevalet est vide et le portrait retourné contre la cheminée, comme pour souligner la double absence de Schön, le commanditaire du tableau, assassiné par le modèle, et de Lulu à la suite de son arrestation, de son procès et de son emprisonnement. Ce sera d'ailleurs la première question de Lulu à son retour: «Où est donc mon tableau? $\|^{47}$, montrant à quel point elle s'est approprié sa représentation au point de la considérer elle-même comme son propre double: «Tu ne l'as pas regardé pendant que j'étais absente ? ${ }^{48}$, demande-t-elle à Alwa. Tout cela, notons-le, alors que Lulu porte les habits de la comtesse Geschwitz dont elle a pris l'identité afin de s'évader de prison. C'est précisément parce qu'elle a pris l'apparence d'une autre que Lulu a besoin de son portrait pour se retrouver telle qu'en elle-même. Alwa, en plaçant le tableau sur le chevalet, énonce un compliment ambigu dans la mesure où il exprime la notion de concurrence: «Tu peux encore rivaliser avec ton portrait $~_{49}$. La réponse de Lulu se montre à la hauteur de cette provocation, lorsqu'elle lui rappelle, au moment du baiser, qu'il devient le rival de son père mort, assassiné par elle (« Du calme !... J'ai assassiné ton père ») ${ }^{50}$.

\section{L'interchangeabilité de l'art et de la réalité}

Au début de l'acte III (un vaste salon orné de stucs blancs), le tableau se trouve encastré dans le mur dans un mince cadre doré, au-dessus d'une commode rococo, presque inaperçu - en tout cas, non évoqué pendant cette longue scène, sauf à la fin, où le 
groom, dans les vêtements de Lulu, reste immobile devant lui. C'est là que le commissaire de police croit pouvoir l'arrêter, le prenant pour Lulu. L'épisode redouble le pouvoir d'illusion du déguisement, de la peinture et de l'art en général, la confusion entre l'original et la copie. Dans la scène 2, le portrait, absent de la chambre londonienne, est rapporté sous sa forme la plus réduite, en tant que rouleau de toile précieusement conservé par la comtesse Geschwitz en dépit de son dénuement extrême et des douze schillings que lui en aurait donnés un chiffonnier. Les réactions divergentes d'Alwa et de Lulu jettent une lumière nouvelle sur ce portrait:

Alwa (saisi d'émotion): Mon Dieu, mais c'est le portrait de Lulu !

Lulu (comme dans un cri): Mon portrait! Hors de ma vue ! Jetez-le par la fenêtre ! $^{51}$

Si Lulu refuse de vivre dans la contemplation d'un passé révolu, si elle rejette à présent ce qui lui rappelle à la fois les séductions involontaires, les morts, les périodes d'aisance matérielle et son crime, Alwa y retrouve une vision cohérente de son parcours, la naissance de son amour pour Lulu lors de la visite initiale - et initiatique - dans l'atelier du peintre, lorsqu'il comparait Lulu avec son portrait. Et c'est à cet endroit qu'Alwa prononce les paroles que le célèbre écrivain et essayiste viennois Karl Kraus (1874-1936) avait citées pour montrer et défendre le rôle social joué par la pièce de Wedekind:

Que celui qui se sent en sécurité dans sa situation bourgeoise devant ces lèvres charnues et éclatantes, devant ces grands yeux pleins d'innocence, devant ce corps rose et généreux... que celui-là nous jette la première pierre ${ }^{52}$.

Kraus, citant le passage correspondant du dernier acte de La Boite de Pandore de Wedekind, le commentait ainsi: "Ces paroles prononcées en face du portrait de la femme qui s'est transformée en destructrice du monde après avoir été détruite par tous, constituent la toile de fond du monde du poète Frank Wedekind. Un monde dans lequel la femme, si elle doit aller jusqu'au bout de son accomplissement esthétique, ne serait pas condamnée à retirer à l'homme le poids de sa responsabilité morale. La prise de conscience de l'abîme tragique qui existe entre des lèvres éclatantes et une condition bourgeoise est peut-être aujourd'hui la seule qui soit digne d'un dramaturge. $»^{53}$

Ce portrait qui rappelle à Alwa les raisons de sa déchéance, ce portrait qui met en danger tout l'édifice social bourgeois, qui confronte l'homme en tant que produit d'une culture et d'une société à un éternel féminin conçu comme innocente sensualité, étrangère aux calculs, transactions et compromissions, rappelle a contrario la fonction du portrait de Dorian Gray dans la nouvelle d'Oscar Wilde (1891) ${ }^{54}$. De manière inversée, c'est chez Wilde le portrait qui se couvre des stigmates de la corruption et de la déchéance de Dorian Gray dont le visage demeure immaculé et éternellement jeune, jusqu'à l'autodestruction finale, geste ultime que Lulu partage en quelque sorte avec lui. Le point commun est celui de l'interchangeabilité de l'art et de la réalité, thème récurrent dans la littérature fin-de-siècle.

\section{De l'instrumentalisation de l'art à l'autonomie retrouvée}

On notera cependant que le portrait est instrumentalisé à nouveau, à l'initiative de Schigolch : «Il faut le clouer au mur, pour notre clientèle »" Miroir aux alouettes, le tableau recouvre le pouvoir de séduction qui fut celui de Lulu. Aussitôt accroché au mur à l'aide de deux clous qu'Alwa enfonce à l'aide de sa botte, d'un coup de talon comme on écrase un insecte - , la toile rescapée - dont on souligne le mauvais état - 
devient, dans une ironie appuyée, la parure du taudis (Schigolch: « Tout l'appartement prend une apparence plus élégante $\left.{ }^{56}\right)$. Il n'est pas indifférent qu'autour de cette toile Geschwitz et Lulu répètent quasiment les mêmes paroles que dans la première scène de l'acte II. Mais cette fois, c'est Schigolch qui donne une réponse plus crue, plus explicite que celle de Schön à la question de la comtesse sur le destin du peintre.

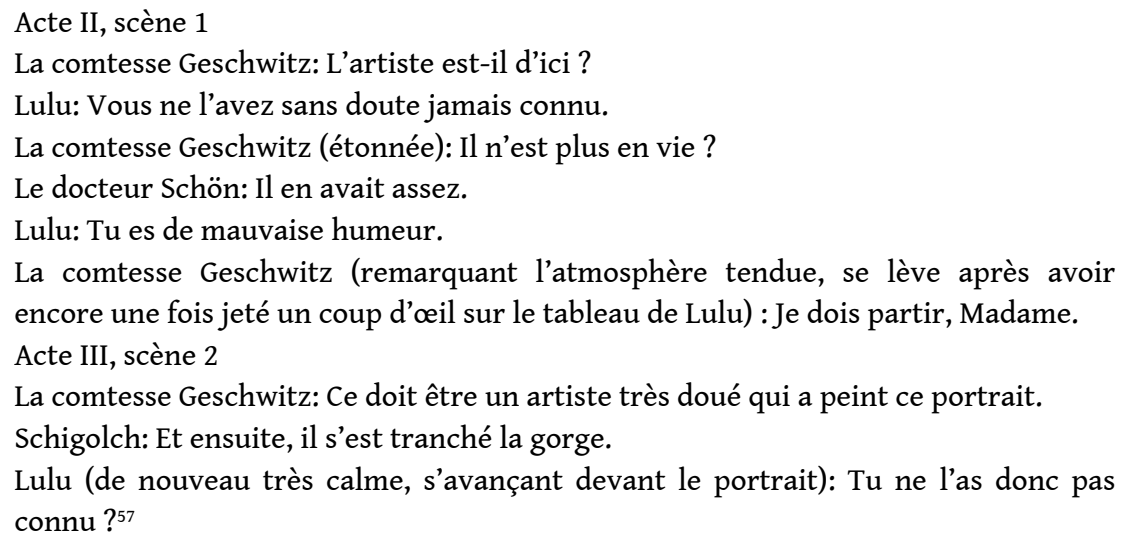

Tandis que Schigolch et Alwa évoquent la beauté passée de Lulu et la persistance de son charme malgré la perte de sa jeunesse, Lulu décide d'en finir: «Je vais me tuer. Je n'en peux plus ici. ${ }^{58} C^{\prime}$ est bien d'un suicide par procuration qu'il s'agit à la fin de l'opéra: de la même manière que Schön avait décidé de mourir, Lulu n'étant dès lors que l'instrument de sa mort, Lulu mourra sous les coups de Jack l'Éventreur parce qu'elle en a décidé ainsi.

31 Lorsque Schigolch reste seul avec Alwa après le départ des deux femmes qui se rendent dans la rue, il regarde le tableau avant de prononcer ces paroles énigmatiques, dont on ne sait pas d'emblée si elles se rapportent à Lulu ou à la comtesse, mais qui semblent bien définir Lulu: «Elle ne peut pas vivre de l'amour parce que sa vie même est amour. $\aleph^{59}$ La phrase exprime une critique de la confusion des valeurs qui conduit à monnayer ce qui par définition est inestimable.

De retour de la rue, la comtesse Geschwitz songe au suicide puis s'adresse au portrait de Lulu dont elle implore la pitié. On peut donc supposer que c'est au portrait encore qu'elle s'adresse à la fin lorsqu'elle s'exclame, avant de mourir (elle aussi, comme Lulu, avait décidé que l'instant de sa mort était arrivé): « Lulu ! Mon ange ! Montre-toi encore une fois! Je suis auprès de toi! Je reste auprès de toi! Pour l'éternité ! ${ }^{60}$ Dépourvu de cadre, détaché de l'ordonnancement d'un atelier, d'un salon ou d'une salle de réception, le portrait recouvre son autonomie et sa plénitude de vérité.

Dans un essai de 1918, Le Problème du portrait ${ }^{61}$, Georg Simmel avait montré que l'art, même s'il n'est que le reflet de l'énergie vitale de la vie réelle, est le lieu d'une expression stable du sens et de l'harmonie, détachée des contingences de l'existence humaine ${ }^{62}$.

\section{Crise du sens: Schopenhauer ou Nietzsche?}

Ultime confusion? Est-ce l'aboutissement d'une conception schopenhauerienne, liée à un pessimisme dénonçant les illusions humaines, concevant le monde comme représentation, conduisant inéluctablement du naturalisme au nihilisme? L'histoire de l'humanité dès lors est vaine, autant que la quête du sens. Mais on peut aussi y voir une 
prise de position nietzschéenne, dans la mesure où "l'éternité » évoquée par la Comtesse Geschwitz s'apparente davantage à un éternel retour qu'à une conception religieuse. S'il est vrai que Wedekind a conçu l'idée d'écrire La Boîte de Pandore en apprenant les meurtres en série de Jack l'Éventreur, la conception cadre de sa pièce serait donc celle de la mise à mort et du dépeçage, voire de l'opération chirurgicale extrême. En même temps, on peut y voir une nouvelle métaphore: c'est la mise à nu des tripes de Lulu, qui, au sens littéral, montre ce qu'elle a dans le ventre. C'est l'aboutissement ultime de la dénudation totale, au-delà de toute apparence. La bestialité de Jack l'Éventreur renvoie à la ménagerie du Prologue, les instincts non domestiqués prennent leur revanche contre un siècle décadent.

La pantomime Lulu, dont Wedekind vit la reprise à Paris en 1892, mettait en scène, aux côtés de la clownesse Lulu, son amoureux le clown «Schopenhauer (Arthur), Officier d'Académie, savant burlesque » et «Arlequin, gommeux » dont s'éprend Lulu. Lors de la création de ce spectacle en 1888, l'allusion à l'actualité de la traduction française par Auguste Burdeau, la même année, du Monde comme Volonté et comme Représentation (1818), texte fondamental du philosophe Schopenhauer (1788-1860), était transparente: la volonté de dérision, de moquerie vis-à-vis d'un public français qui s'était entiché du pessimisme schopenhauerien était manifeste. Mais Wedekind y trouve la confirmation de sa conception de l'être: la vérité n'existe que dans l'instinct, elle est travestie, occultée par les conventions de la société. Le vouloir-vivre, pour utiliser les catégories schopenhaueriennes, est la racine de tous les maux, dans un cycle absurde et sans fin dont seul l'art peut nous affranchir. En Lulu, Wedekind ne voit pas un clown mais une tragédienne ${ }^{63}$.

Schopenhauer considérait, dans Le Monde comme Volonté et comme Représentation ${ }^{64}$, que le monde des idées et de la création était celui de l'homme par opposition au monde de la sensualité et de la séduction qui serait celui de la femme. La création n'était possible pour l'homme selon lui que par l'affranchissement des désirs, le détachement par rapport à la femme censée le détourner de ses préoccupations intellectuelles et artistiques. Annonçant ainsi la thèse freudienne de la sublimation, Schopenhauer autorise des extrapolations: l'absence de possibilité de création conduirait à la stérilité culturelle et à la décadence ${ }^{65}$. Lulu ne contamine-t-elle pas Alwa après avoir été ellemême contaminée par le Marquis sans pour autant être atteinte, comme Alwa le dévoile à Schigolch (III, 2) ? Dans L'esprit de la terre, la pièce de Wedekind, Schön comparait Lulu à une "contagion incurable $»^{66}$. Si la philosophie de Schopenhauer conduit in fine, par la pitié, à la résignation ${ }^{67}$, la pensée de Nietzsche, à qui Wedekind doit beaucoup, exprime l'affirmation de la vie. C'est dans le vitalisme nietzschéen que Berg (comme Wedekind) peut trouver une justification à la représentation du laid, qui fait partie de la vie au même titre que le beau. Contre l'exclusivité de la belle apparence, le laid est nécessaire à l'expression de la vérité.

On peut considérer qu'avec Wozzeck (1925) et Lulu, une esthétique de la laideur se constitue sur la scène lyrique de langue allemande, fondée, dans les livrets, sur une approche philosophique et littéraire que conforte et renouvelle l'écriture musicale exploitant entre autres les ressources de la dissonance, de l'atonalité et du dodécaphonisme en lien étroit avec l'expression et l'interprétation du texte. La problématique de la laideur à l'opéra entre en contradiction avec l'art du beau chant ${ }^{68}$, dont la prédominance est remise en cause, en même temps que l'image idéalisée de la femme, depuis Salomé (1905) et Elektra (1909) de Richard Strauss (1864-1949). 
Parallèlement à cette émancipation des figures féminines, la place du cri dans la musique, le rapport dialectique qu'entretiennent consonance et dissonance, l'alternance du parlé et du chanté ou leur réunion sous la forme du Sprechgesang ouvrent à l'opéra des perspectives nouvelles qui vont de pair avec l'expression verbale et la représentation théâtrale d'une réalité qui donne à voir et à entendre la laideur autant que la beauté, dans une sorte de réponse à la contradiction perceptible chez Schopenhauer entre la recherche du plaisir esthétique et le pessimisme. La fin sanglante de Lulu doit provoquer chez le spectateur un choc esthétique à la manière expressionniste et conduire à une relecture de l'ensemble de l'opéra dans cette perspective d'une destinée sacrificielle, Lulu étant la victime expiatoire des vices d'une société malade de sa modernité et confrontée à la perte radicale du sens que seule l'œuvre d'art peut tenter de reconstruire par des réseaux nouveaux de signification.

\section{NOTES}

1. Cette contribution est la version modifiée d'un article publié en 2005 dans le programme de l'Opéra National du Rhin: Lulu de Berg. Strasbourg, 2005, p. 46-62, sous le titre « Lulu d'Alban Berg ou la crise de la modernité ». Je remercie Monique Herzog de m'avoir aimablement autorisé à en reprendre ici l'essentiel.

2. Voir à ce sujet l'article de Christian Merlin, «Frank Wedekind à l'opéra. De 'La Boîte de Pandore' à 'Lulu' de Berg ", in L'Avant-Scène Opéra n¹81/182, 1998, p. 172-180, et l'étude de Bernard Banoun, «Lulu d'Alban Berg, opéra de l'apparence ", in Études Germaniques n 60 , janviermars 2005, p. 109-121 (je n'avais pas connaissance de cette étude lorsque j'ai écrit à la même époque la première version de ce texte).

3. Si c'est bien Lulu qui appuie par cinq fois sur la détente, c'est le docteur Schön qui lui a remis le revolver, tentant de la convaincre de se suicider. Mais c'est en définitive, de la part de Schön qui se refuse à envisager le divorce "parce qu'il ne fait plus qu'un » avec Lulu, un suicide par procuration. Lulu n'apparaît pas plus responsable de ce meurtre que de celui de la femme de Schön, dont elle dit à Alwa qu'elle l'a empoisonnée (II, 1), formule à comprendre comme une image (au moment où elle veut dissuader Alwa de tomber amoureux d'elle): sa seule présence au foyer des Schön peut avoir été la cause indirecte de la mort de Madame Schön, dont la nature féminine, dans la société ici mise en accusation, était niée par sa fonction d'épouse et de mère, contrairement à Lulu, jeune et libre, incarnant la «figure originale » de la féminité selon les propos du dompteur dans le Prologue.

4. » Ich habe nie in der Welt etwas anderes scheinen wollen, als wofür man mich genommen hat. Und man hat mich nie in der Welt für etwas anderes genommen, als was ich bin. » Je cite le texte allemand d'après le livret publié dans L'Avant-Scène Opéra $n^{\circ} 181 / 182$, 1998, p.19-151. Sauf indication contraire, la traduction française citée est celle de Bernard Banoun présentée dans ce même ouvrage. Ici, traduction légèrement modifiée.

5. Cette série de questions et de réponses évoque un anti-catéchisme: «- Kannst du die Wahrheit sagen ? - Ich weiß es nicht. - Glaubst du an einen Schöpfer ? - Ich weiß es nicht. - Kannst du bei etwas schwören. - Ich weiß es nicht. - Woran glaubst du denn ? - Ich weiß es nicht. [...] - Hast du denn keine Seele ? - Ich weiß es nicht. - Hast du schon einmal geliebt ? - Ich weiß es nicht. - Sie weiß es nicht. - Ich weiß es nicht. " Livret in L'Avant-Scène Opéra n 181/182, 1998, p. 31. 
6. Sur lui, voir notamment François Léger, La Pensée de Georg Simmel. Contribution à l'histoire des idées en Allemagne au début du XXe siècle, Paris, Kimé, 1989. Ingeburg Lachaussée a soutenu en 2003 une thèse de doctorat (Paris IV, dir. J.-M. Valentin) intitulée Georg Simmel. Argent et philosophie.

7. Voir l'introduction et la traduction française de Jean-Louis Vieillard-Baron: Georg Simmel : Philosophie de la modernité. Paris : Payot, 1989.

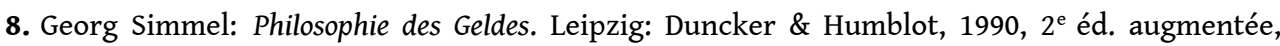
1907 ; Grundfragen der Soziologie (Individuum und Gesellschaft). Berlin und Leipzig: Göschen, 1917; Zur Philosophie der Kunst. Philosophische und kunstphilosophische Aufsätze. Potsdam: Kiepenheuer, 1922. Voir également la traduction française par Sabine Cornille et Philippe Ivernel d'une série d'essais de Georg Simmel sous le titre : Philosophie de l'amour. Paris : Payot Rivages, 1991.

9. Voir F. Léger, op. cit. (note 6), p. 303 et passim.

10. Voir F. Léger, op. cit. (note 6), notamment p. 80-96.

11. » Kunstgewerblerin : Stilkleid », Livret in L'Avant-Scène Opéra n 181/182, 1998, p. 109.

12. » Ich liebe eigentlich mehr die Akrobaten », ibid., p. 111.

13. » Marquis : - Wie kommt es, daß man eine niedliche, kleine Prinzessin heute zum ersten Mal sieht?»Ibid., p. 111.

14. » Ich tauge nicht für diesen Beruf », ibid., p. 115. C'est ce que répond Lulu au Marquis qui veut la prostituer, ajoutant: «Par bonheur, j'ai passé trois mois à l'hôpital sans voir un homme. J'ai alors ouvert les yeux sur moi-même et me suis reconnue pour celle que j'étais. » (« Dann lag ich glücklicherweise drei Monate im Krankenhaus, ohne eine Mann zu Gesicht zu bekommen. In jener Zeit gingen mir die Augen über mich auf, und ich erkannte mich.»)

15. "Ich lasse mich immatrikulieren. Ich muß für Frauenrechte kämpfen, Jurisprudenz studieren. » Livret p. 151.

16. » Du hast eine halbe Million geheiratet... » Livret p. 43 et 45 . Schön est interrompu par le peintre lorsqu'il tente de prononcer la phrase une quatrième fois (Livret p. 47). La mélodie souligne ces répétitions.

17. » LULU: - Bleib! Es ist ja niemand zuhaus! Wir machen ganz einfach nicht auf! DER MALER: Vielleicht ist es aber der Kunsthändler... » Livret p. 35.

18. » LULU: - Ich würde so gerne mit dir ausfahren. DR. sCHÖN: - Du weißt, daß ich heute auf die Börse muß. » Livret p. 71.

19. Cf. Sophie Basch : Romans de cirque. Paris : Robert Laffont, 2002.

20. » Ihr stolzen Herren, Ihr lebenslust'gen Frauen »; «Die unbeseelte Kreatur [...] / Gebändigt durch das menschliche Genie. » Livret p. 19.

21. Comme l'écrit Sophie Basch, op. cit., p. 598.

22. » Lulu ! Mein Engel! » Livret p. 151.

23. » Du Würgengel!» II, 1 (Livret p. 87). Lulu souligne quant à elle la nature hybride de la comtessse Geschwitz: «Tu n'es pas un être humain comme les autres. Il manquait de la matière pour faire un homme, et pour une femme tu as trop de cervelle, voilà pourquoi tu es folle » III, 1(« Du bist kein Menschenkind wie die andern. Für einen Mann war der Stoff nicht ausreichend. Und zum Weib hast du zu viel Hirn in deinen Schädel bekommen. Darum bist du verrückt!»). Livret p. 121.

24. » Die süße Unschuld - meinen größten Schatz!» Livret p. 20, traduction légèrement modifiée.

25. » Sie ward geschaffen, Unheil anzustiften, / Zu locken, zu verführen, zu vergiften - / und zu morden - ohne daß es einer spürt. », ibid., traduction légèrement modifiée.

26. » Porte-la à sa place » (« Trag sie an ihren Platz. ») Ibid.

27. » Und nun bleibt noch das Beste zu erwähnen: / Mein Schädel zwischen eines Raubtiers Zähnen. » Livret p. 20.

28. Bernard Banoun montre que ce film est «le noyau structurel de l'œuvre », op. cit. (note 1), p. 113. 
29. Alban Berg manifestait un grand intérêt pour l'essor contemporain du cinéma.

30. Dans le texte de Wedekind, Alwa n'est pas compositeur, mais écrivain et dramaturge.

31. » Darf ich eintreten? » Livret p. 21.

32. Voir par exemple Dominique Jameux, « Abécédaire Lulu » dans L'Avant-Scène Opéra 181/182, op.cit., p.154, qui rappelle aussi qu'Alwa est "présent musicalement par sa série dans le Prologue » (voir le commentaire musical de Gérard Condé, ibid., p. 18, montrant l'utilisation de la série d'Alwa dans la deuxième strophe de la harangue du dompteur).

33. » Der Maler vor der Staffelei, malend. » Livret p. 21.

34. " Du hast kein Recht, uns durch Miaun und Pfauchen / Die Urgestalt des Weibes zu verstauchen. » Livret p. 20.

35. On peut aussi y voir, de la part d'Alban Berg, un hommage au Pierrot lunaire (1912) de son maître et ami Arnold Schoenberg, œuvre qui fit scandale en son temps et qui, par l'utilisation du Sprechgesang, rapprochait le chant de la vérité de la parole. Mais le motif du portrait de Lulu en Pierrot se trouve déjà dans la pièce L'Esprit de la terre de Wedekind.

36. » Ich mich verstellen? Das hatt'ich niemals nötig. » Livret p. 28.

37. » Er sieht nichts; er sieht mich nicht und sich nicht. Er ist blind, blind, blind... » Livret p. 39 et p. 40 .

38. » DER MALER : - Ich liebe dich, Nelly ! LuLu: - Ich heiße nicht Nelly, ich heiße Lulu. DER MALER : Ich werde dich Eva nennen. » Livret p. 27.

39. » DER MALER : - Von wem sprichst du denn? DR. SCHÖN : - Von deiner Frau ! M. : - Von Eva ? s. : Ich nannte sie Mignon. M. : - Ich meinte, sie hieße Nelly. s. : - So nannte sie Dr. Goll. M. : - Ich nannte sie Eva... s. : - Wie sie eigentlich heißt, weiß ich nicht. » Livret p. 45.

40. » Daß du mich Lulu nennst ! [...] Ich heiße seit Menschengedenken nicht mehr Lulu. » Livret p. 38.

41. » Sehr eleganter Salon. " « An einer Wand über dem Kamin ein prachtvoller Brokatrahmen : Lulus Bild als Pierrot. » Livret p. 32.

42. » Unsichtbar hinter der spanischen Wand. » Livret p. 53.

43. » Über die ließe sich freilich eine interessante Oper schreiben. (vor dem Plakat stehend) Erste Szene: Der Medizinalrat... Schon faul! (Langhaltendes, stark gedämpftes Klatschen und Bravorufen wird von außen hörbar.) Das tobt wie in einer Menagerie, wenn das Futter vor dem Käfig erscheint. (wieder beim Bild) Zweite Szene: Der Maler... Noch unmöglicher! - Dritte Szene: Sollte es wirklich so weitergehn? » Livret p. 55.

44. Voir les didascalies du livret p. 56.

45. » Lulus Bild als Pierrot in antiquisiertem Goldrahmen. » Livret p. 68.

46. » Hier sind Sie wie ein Märchen. » Livret p. 69, traduction modifiée.

47. » Wo ist denn mein Bild?» Livret p. 104.

48. » Du hast es nicht angesehn, während ich fort war? » Ibid.

49. » Mit deinem Bild kannst du es immer noch aufnehmen. » Livret p. 105.

50. » Ruhig ! - Ich habe deinen Vater erschossen. » Livret p. 106.

51. » ALWA: - Mein Gott, das ist ja Lulus Bild. LULU: - Mein Bild! Mir aus den Augen! Werft es zum Fenster hinaus! » Livret p. 141 (traduction modifiée).

52. » Wer sich vor diesen blühenden, schwellenden Lippen, vor diesen großen, unschuldsvollen Kinderaugen, vor diesen rosigweißen, strotzenden Körper in einer bürgerlichen Stellung sicher fühlt - der werfe den ersten Stein auf uns. » Livret p. 141.

53. » Diese Worte, vor dem Bilde des Weibes gesprochen, das zur Allzerstörerin wurde, weil es von allen zerstört war, umspannen die Welt des Dichters Frank Wedekind. Eine Welt, in der die Frau, soll sie ihrer ästhetischen Vollendung reifen, nicht verflucht ist, dem Mann das Kreuz sittlicher Verantwortung abzunehmen. Die Erkenntnis, welche die tragische Kluft zwischen blühenden Lippen und bürgerlichen Stellungen begreift, mag heute vielleicht die einzige sein, die eines Dramatikers wert ist. » Karl Kraus: Werke, Bd. 6 « Literatur und Lüge ». Munich: Kösel, 1958, 
p. 10. Traduction française d'Annick Carlier, in L'Avant-Scène Opéra $\mathrm{n}^{\circ} 181 / 182$, 1998, p. 170. Il s'agit du texte d'une conférence prononcée par Kraus à l'occasion d'une représentation privée de la pièce le 19 mai 1905, à laquelle Alban Berg assista.

54. Ce rapprochement est également effectué par Bernard Banoun, op. cit. (note 1), p. 114.

55. » Man muß es annageln, für unsere Kundschaft. » Livret p. 141 (traduction modifiée).

56. » Das ganze Appartement bekommt ein eleganteres Aussehen. » Ibid., traduction modifiée.

57. » G. : - Ist es von einem Hiesigen ? L.: - Sie werden ihn kaum gekannt haben. G.: - Er lebt nicht mehr? S.: - Er hatte genug. L.: - Du bist verstimmt. G. (die die unbehagliche Stimmung bemerkt, erhebt sich, nachdem sie nochmals Lulus Bild mit dem Blick gestreift hat): - Ich muß gehn, Frau Doktor. » (II, 1) Livret p. 69. « G.: - Es muß ein sehr begabter Künstler gewesen sein, der das Bild gemalt hat. S.: - Und später hat er sich den Hals abgeschnitten. L.: - Hast du ihn denn nicht gekannt? » (III, 2) Livret p. 141.

58. » Ich bring' mich um. Ich halt's hier nicht mehr aus. » Livret p. 143.

59. » Die kann von der Liebe nicht leben, weil ihr Leben die Liebe ist. » Livret p. 144.

60. » Lulu! Mein Engel! Laß dich noch einmal sehn! Ich bin dir nah! Bleibe dir nah! In Ewigkeit! » Livret p. 151.

61. In Die neue Rundschau, XXIX. Jg. der freien Bühne, 1918, Bd. 2 ( = Heft 10 vom Oktober 1918), S. 1336-1344. Le texte est disponible sur <http ://socio.ch/sim/verschiedenes/1918/portrait.htm> [page consultée le 10 mars 2008].

62. L'essai de Simmel se termine par ces mots: «Denn da schließlich doch auch sie [ = die Kunst] aus dem Leben kommt, aus seinem Pulsschlag die Kräfte ihrer Entwicklung zieht, so ist die Harmonie, die die Dinge in ihrem Spiegel finden, so partiell sie sein mag, uns eine Ahnung und ein Pfand dafür, daß die Elemente des Lebens im allertiefsten Grunde auch ihrer Wirklichkeit doch vielleicht nicht so hoffnungslos gleichgültig und gegensätzlich auseinander liegen, wie das Leben selbst uns so oft glauben machen will. »

63. Cf. Sophie Basch, op. cit. (note 7), p. 587-598.

64. Arthur Schopenhauer: Die Welt als Wille und Vorstellung (1819-1844).

65. Sur ces points et sur le contexte de la modernité, voir Michel Pollak: Vienne 1900. Une identité blessée. Paris : Gallimard/Julliard, 1984 (coll. Archives).

66. Frank Wedekind, Erdgeist, acte IV, scène 8: «Schön : - [...] Du haftest mir als unheilbare Seuche an, an der ich bis in mein Grab meine Lebenszüge verächzen soll. »

67. Pour Schopenhauer, le fondement de la morale est la pitié: dans la souffrance de l'autre je vois ma propre souffrance.

68. Bernard Banoun note à ce sujet: « Conformément à une idée directrice de la pensée musicale d'Adorno, la musique de l'époque moderne ne peut être 'belle'. Seule est en concordance avec l'époque une musique capable de dire la perte ou la nostalgie de la beauté et du bonheur, et non leur simple inexistence », op. cit. (note 1), p. 118.

\section{RÉSUMÉS}

Le livret de l'opéra Lulu d'Alban Berg illustre la crise du sens au sein de la modernité par la métaphore d'un monde autodestructeur, par la remise en cause du statut et de l'image de la femme, par une interrogation sur le rôle de l'argent et sur celui de l'art. La parenté du personnage de Lulu avec celui de Lola Fröhlich dans le roman Professor Unrat (1905) de Heinrich 
Mann (rendu célèbre par le film de Sternberg, L'ange bleu) ainsi que le rapprochement du contenu idéel du livret avec les analyses du philosophe et sociologue contemporain Georg Simmel montrent comment Berg a mis en valeur l'actualité des thèmes traités par Wedekind dans les pièces qui sont à l'origine du livret de l'opéra, tout autant que la réception de la pensée de Schopenhauer et de Nietzsche. De Wedekind, que Heinrich Mann désignait comme un «pionnier en littérature ", à Alban Berg, qui est incontestablement un pionnier dans le domaine musical, la problématique de l'art qui se trouve au centre du texte constitue aussi le noyau de l'écriture littéraire, musicale et scénique, dans une manière de mise en abyme liée à la dénonciation de la confusion des valeurs, du primat de l'apparence et du faux-semblant, du pouvoir et de la violence. Cette exposition kaléidoscopique de contradictions présente une société malade de sa modernité, confrontée à la perte radicale du sens que l'œuvre d'art - en l'occurrence le livret d'opéra - tente de réinvestir en créant de nouveaux réseaux de signification.

Das Libretto von Alban Bergs Lulu dokumentiert die Sinnkrise innerhalb der Modernität durch die Metapher einer selbstzerstörerischen Welt, durch die Infragestellung des Status und des Bildes der Frau, durch die Frage nach der Rolle des Geldes und nach der Rolle der Kunst. Die Verwandtschaft der Figur von Lulu mit der von Lola Fröhlich in Heinrich Manns Roman Professor Unrat (1905), den Sternbergs Film Der blaue Engel berühmt machte, sowie der Vergleich des Ideengehalts des Librettos mit den Analysen des Philosophen und Soziologen Georg Simmel zeigen, wie Berg neben der Rezeption von Schopenhauer und Nietzsche die Aktualität der Themen hervorgehoben hat, die Wedekind in den Dramen behandelte, die die Grundlage für das Libretto lieferten. Von Wedekind - nach Heinrich Mann ein «Pionier in Literatur » - bis Alban Berg, der unstreitig ein Pionier im Bereich der Oper war, bildet die Problematik der Kunst, die im Text zentral ist, zudem den Kern des literarischen, musikalischen und szenischen Schaffens in einer Art mise-en-abyme, die mit der Kritik an der Verwechslung der Werte, an dem Primat des Scheins und der Verstellung, der Macht und der Gewalt verbunden ist. Diese kaleidoskopische Darstellung von Gegensprüchen zeigt uns eine an ihrer Modernität kranke Gesellschaft, die mit dem radikalen Verlust des Sinns konfrontiert ist, den das Kunstwerk - hier das Libretto - durch neue Vernetzungen wieder herzustellen versucht.

\section{INDEX}

oeuvrecitee Lulu, Professor Unrat, L'Ange bleu

Mots-clés : modernité

\section{AUTEURS}

\section{FABRICE MALKANI}

Université Lumière Lyon 2 